\title{
Soluble Programmed Death-Ligand 1 (sPD-L1) is Elevated in Aggressive Prostate Cancer Disease Among African Men
}

\author{
Paul Katongole (D) - Obondo J. Sande · Steven J. Reynolds • \\ Moses Joloba · Henry Kajumbula · Samuel Kalungi · Kenneth Ssebambulidde • \\ Cynthia Nakimuli · Maxine Atuheirwe · Jackson Orem • \\ Nixon Niyonzima
}

Received: December 10, 2021 / Accepted: January 13, 2022 / Published online: February 7, 2022

(c) The Author(s) 2022

\section{ABSTRACT}

Introduction: Programmed death 1 (PD-1)/ programmed death-ligand 1 (PD-L1)-targeted immunotherapies have become a new mode of treatment for several tumours; however, there is limited evidence on the expression and

Supplementary Information The online version contains supplementary material available at https:// doi.org/10.1007/s40487-022-00184-6.

P. Katongole $(\bowtie) \cdot$ H. Kajumbula

Department of Medical Microbiology, College of

Health Sciences Makerere University, Kampala,

Uganda

e-mail: paul.katongole@gmail.com

O. J. Sande · M. Joloba · C. Nakimuli

Department of Immunology and Molecular Biology,

College of Health Sciences Makerere University,

Kampala, Uganda

S. Kalungi

Pathology Department, Mulago National Referral

Hospital, Kampala, Uganda

K. Ssebambulidde $\cdot$ M. Atuheirwe

Infectious Diseases Institute, College of Health

Sciences, Makerere University, Kampala, Uganda

J. Orem · N. Niyonzima

Uganda Cancer Institute, Kampala, Uganda

S. J. Reynolds

Division of Intramural Research, National Institute of Allergy and Infectious Diseases, National

Institutes of Health, Bethesda, MD, USA prognostic value of PD-1/PD-L1 in prostate cancer, especially in African men.

Methods: Plasma concentrations of PD-L1/PD-1 were assessed using enzyme-linked immunosorbent assay in patients with prostate cancer and normal healthy controls at the Uganda Cancer Institute. The associations between plasma PD-L1/PD-1 concentration levels and serum prostate-specific antigen (PSA) levels, Gleason scores, age, and body mass index (BMI) were determined.

Results: We found significant differences in the median plasma concentrations of PD-L1 and PD-1 immune checkpoint molecules between prostate cancer cases and normal healthy controls of 0.285 vs $0.035(p=0.001)$ and 0.596 vs $0.355(p=0.017)$, respectively. We found no significant association between age, serum PSA levels, BMI and Gleason scores, and PD-1 among patients with prostate cancer and controls. However, elevated levels of PD-L1 were significantly associated with higher Gleason scores among patients with prostate cancer $(p=$ $0.014)$.

Conclusions: Elevated PD-L1 levels were statistically significantly linked to high Gleason scores. These results may guide clinicians in assessing the prognosis of patients individually and selecting patients who will be suitable candidates for anti-PD-L1 immunotherapy.

Keywords: Immune checkpoint inhibitors; Immunotherapy; Prostate cancer; sPD-1; sPD-L1 


\section{Key Summary Points}

Prostate cancer is the second most frequently diagnosed cancer in men worldwide.

Men of African ancestry suffer disproportionately from prostate cancer at a rate almost twice as high as men of European origin.

Programmed death 1 (PD-1)/programmed death-ligand 1 (PD-L1)-targeted immunotherapies are promising treatment modalities for several tumours; however, there is limited evidence on the expression and prognostic value in prostate cancer, especially among African men.

We hypothesized that soluble programmed death 1 (sPD-

1)/soluble programmed death-ligand 1 (sPD-L1) levels would be associated with prostate cancer disease aggression among men with prostate cancer in Uganda.

We found that elevated levels of sPD-L1 were significantly associated with higher Gleason scores among patients with prostate cancer $(p<0.05)$.

This study sheds more light on the role of sPD-L1 as a potential biomarker for prostate cancer patients, particularly African men.

\section{INTRODUCTION}

Prostate cancer is the second most frequently diagnosed cancer in men worldwide, accounting for approximately $15 \%$ of all new cancer diagnoses in men [1]. In 2020, the Global Cancer Observatory (GLOBOCAN) report noted an annual incidence of 1.4 million prostate cancer cases worldwide, with approximately 375,304 deaths [2]. Reports indicate that men of African ancestry suffer disproportionately from prostate cancer at a rate almost twice as high as men of European origin, irrespective of new treatment modalities [3-5].

The mainstay of treatment for advanced prostate cancer remains androgen deprivation therapy (ADT) [6]. Among patients with metastatic prostate cancer, various treatment options for metastatic castration-resistant prostate cancer (mCRPC), such as hormonal therapy, chemotherapy, and radiopharmaceuticals, have shown significantly increased overall survival (OS). Still, these patients ultimately develop resistance $[7,8]$.

Over the last decade, the introduction of immunotherapy, which works through the augmentation of the immune response against cancers, has yielded promising results and has significantly changed the treatment landscape owing to its efficacy and minimal side effects $[9,10]$. Thus, immune checkpoint inhibitors have emerged as a complementary treatment arm in cancer clinical care and research $[11,12]$. The co-inhibitory receptor programmed death 1 (PD-1) is expressed on activated T cells and $\mathrm{B}$ cells. Upon binding of PD-1 to its ligand, PD-L1, which is expressed on macrophages, dendritic cells and some tumour cells, T cell activation is downregulated via inhibition of CD28 signalling [13]. In addition to expression on cell membranes, several extracellular (soluble) forms of PD-L1 (sPD-L1), including spliced variants and proteolytic cleavage forms, have been reported in various types of cancer [14]. sPD-L1 can bind to PD-1 on T cells and suppress antitumour immunity, thus facilitating tumour growth. Blocking the PD-1/PD-L1 interaction using immune checkpoint inhibitors has been shown to reduce tumour growth and increase OS of patients [15].

Anti-PD-1 antibody immunotherapy (e.g. nivolumab) and anti-PD-L1 antibody (e.g. atezolizumab) have demonstrated improved response rates and OS among patients with melanoma and renal cell carcinoma, respectively $[16,17]$.

Ongoing clinical trials show that immunotherapy might provide a promising approach for the future treatment of prostate cancer [18]. Studies indicate that PD-L1 expression is upregulated in prostate cancer tissues 
compared to paired benign prostate tissues. Similarly, high levels of PD-L1 expression have been shown to correlate positively with high Gleason scores and androgen receptor expression in patients with aggressive primary prostate cancer [19-21].

This study assessed the circulating levels of immune checkpoint molecules, PD-1 and PDL1, among prostate cancer patients and normal healthy controls and their association with age, body mass index (BMI), serum prostate-specific antigen (PSA), and Gleason scores at the Uganda Cancer Institute.

\section{METHODS}

\section{Study Population}

This was a case-control study in which 86 men, including 57 prostate cancer patients on ADT and 29 normal healthy controls, were recruited from January 2020 to June 2020 at the Uganda Cancer Institute, Kampala, Uganda. All study participants were aged 40 years and older. Controls were men with no history of prostate cancer and with a PSA of less than $4 \mathrm{ng} / \mathrm{ml}$. Cases were men with a histological diagnosis of prostate cancer who were receiving ADT.

\section{Study Procedures}

A predesigned questionnaire was used to obtain demographic data and the medical history of study participants. Six millilitres of blood was collected from each study participant in an ethylenediaminetetraacetate (EDTA) anticoagulation tube by a trained study nurse. Blood samples were transported within $1 \mathrm{~h}$ to the laboratory for processing. This study obtained ethical approval from the School of Biomedical Sciences Higher Degrees Research and Ethics Committee (REF: SBS-HDREC-779) and the Uganda National Council for Science and Technology (UNCST). All study participants provided written informed consent before enrolment in the study.

\section{Plasma Collection and Immunoassays}

\section{Separation of Platelet-Poor Plasma}

All laboratory procedures and tests were carried out in the Translational Research Laboratory at the Infectious Diseases Institute, Makerere University, Kampala, Uganda. Upon receipt in the laboratory, whole blood samples were immediately centrifuged at $6^{\circ} \mathrm{C}$ at a speed of $1000 \times g$ for $10 \mathrm{~min}$. The supernatant was collected in a sterile falcon tube and centrifuged again at $1600 \times g$ for $10 \mathrm{~min}$ at $6^{\circ} \mathrm{C}$. The resulting supernatant was then separated into two aliquots and stored at $-80^{\circ} \mathrm{C}$ for later use to perform the immunoassays.

\section{Enzyme-Linked Immunosorbent Assay (ELISA)}

Plasma levels of immune checkpoint regulators were measured in picograms per millilitre, and commercially procured control samples for each analyte were assayed in parallel to ensure good results. Stored plasma samples were retrieved and thawed at $4-8{ }^{\circ} \mathrm{C}$. Immunoassays were performed in duplicate using human checkpoint marker PD-1 and PD-L1 Quantikine ELISA kits (R\&D Systems), following the manufacturer's instructions.

\section{Statistical Analysis}

Categorical variables (demographic and clinical factors) were expressed as absolute numbers and proportions. Continuous variables (plasma concentration of cytokines) were expressed as medians and interquartile ranges. The Mann-Whitney $U$ test was used to compare continuous variables, and Fisher's exact test for comparing categorical variables. The association of immune checkpoint molecules with different ages, Gleason scores, PSA, and BMI was explored using conditional linear regression. All analyses were performed using Stata 16 software. 


\section{RESULTS}

\section{Clinico-Demographic Factors in Prostate Cancer Patients and Controls}

The median age was 70 years in cases and 59 years in the control group. The median BMI was 20.84 in the cases and 19.45 in the control group. Among the study participants, $49.43 \%$ of cases and $68.97 \%$ of controls had normal BMI in the range of 18.5-24.9. The median PSA was $33.01 \mathrm{ng} / \mathrm{ml}$ and $1.8 \mathrm{ng} / \mathrm{ml}$ among cases and controls, respectively. Among the cases, $31.58 \%$ had a PSA of $>100 \mathrm{ng} / \mathrm{ml}$. Of the prostate cancer patients, $43.86 \%$ had advanced prostate cancer, with Gleason scores of 8-10. A full description of clinical and demographics characteristics is provided in Table 1.

\section{Plasma Levels of Immune Checkpoint Molecules}

The median concentrations of PD-1 and PD-L1 were generally higher in cases than in controls. We found significant differences in the plasma concentrations of PD-L1 and PD-1 immune checkpoint molecules between prostate cancer cases and normal healthy controls $(p=0.0101$ and 0.017, respectively), as seen in Fig. 1. We found that high levels of PD-L1 were significantly associated with higher Gleason scores among patients with prostate cancer $(p=0.014)$ (Table 2).

However, the study found no significant association between age, serum PSA levels, BMI and Gleason scores, and PD-1 checkpoint molecules among patients with prostate cancer and controls.

\section{DISCUSSION}

This was the first study to assess the association between sPD-1/PD-L1 levels and prostate cancer clinical characteristics in African populations. The study evaluated the circulating concentrations of immune checkpoint molecules PD-1 and PD-L1 and their association with PSA levels, Gleason scores, age, and BMI among patients with prostate cancer and controls at the Uganda Cancer Institute. Consistent with previous studies, our results suggest a significant association between PD-L1 levels and high Gleason scores, predictive of aggressive tumour behaviour in prostate cancer [20, 22-24].

This finding further suggests that the PD-1/ PD-L1 pathway activation supports the evasion of the antitumour immune response, driving tumour pathogenesis. The probability of antitumour immune response to anti-PD-1/anti-PDL1 antibody therapy is associated with the expression of PD-L1 on the tumour cell surface [24-27].

Different mechanisms by which PD-L1-expressing cells evade $\mathrm{T}$ cell immunity have been hypothesized, namely: inducing (1) apoptosis, (2) T cell energy, or (3) functional exhaustion of $\mathrm{T}$ cells, (4) forming a molecular shield to keep lysis off tumour cells, (5) increasing production of the immunosuppressive cytokine IL-10, and (6) facilitating Treg cell-mediated suppression [28, 29]. Increased expression of PD-L1 on tumour cells has previously been described for several malignancies, including glioblastoma, pancreatic, ovarian, breast, renal, head and neck, oesophageal, and non-small cell lung cancer. PD-L1 [30] expression has also been associated with poor prognosis and adverse clinico-pathological characteristics [21, 31, 32]. A study by Gevensleben et al. (2016) provided early evidence of abundant expression of PD-L1 as a common occurrence in primary prostate cancer and as a negative predictor of biochemical recurrence (BCR)-free survival [23, 33]. Similarly, Heng Li et al. [34] looked at the expression of $\mathrm{PD}-1 / \mathrm{PD}-\mathrm{L} 1$ in a retrospective cohort of men with prostate cancer who received adjuvant hormonal therapy (AHT) after radical prostatectomy (RP). They found moderate to high PD-L1 expression in $49.6 \%$ of primary prostate cancers after radical prostatectomy. In addition, PD-L1 expression was significantly associated with reduced BCR-free survival.

Topalian et al. [35] carried out an immunohistochemical assessment of PD-L1 in pretreatment cancer specimens from 42 patients. They reported that objective response to treatment was seen exclusively in (36\%) PD-L1-positive 
Table 1 Clinical characteristics of prostate cancer patients and controls

\begin{tabular}{|c|c|c|}
\hline Clinical characteristics & Cases $(n=57)$ & Controls $(n=29)$ \\
\hline \multicolumn{3}{|l|}{ Age (years) } \\
\hline Median (IQR) & $70(44-89)$ & $59(44-82)$ \\
\hline$<40$ & $1(1.75 \%)$ & $0(0.00 \%)$ \\
\hline $41-65$ & $17(29.82 \%)$ & $18(62.07 \%)$ \\
\hline $66-75$ & $26(45.61 \%)$ & $6(20.69 \%)$ \\
\hline$>75$ & $13(22.81 \%)$ & $5(17.24 \%)$ \\
\hline \multicolumn{3}{|l|}{ BMI $\left(\mathrm{kg} / \mathrm{m}^{2}\right)$} \\
\hline Median (IQR) & $20.84(16.03-27.61)$ & $19.45(17.22-22.46)$ \\
\hline$<18.5$ & $8(14.04 \%)$ & $9(31.03 \%)$ \\
\hline $18.5-24.9$ & $43(49.43 \%)$ & $20(68.97 \%)$ \\
\hline $25-29.9$ & $6(10.53 \%)$ & $0(0.00 \%)$ \\
\hline 30 and above & $0(0.00 \%)$ & $0(0.00 \%)$ \\
\hline Gleason score & & $\mathrm{N} / \mathrm{A}$ \\
\hline Median (IQR) & $7(6-10)$ & - \\
\hline $6(3+3)$ & $13(22.8 \%)$ & - \\
\hline $7(3+4)$ or $(4+3)$ & $19(33.33 \%)$ & - \\
\hline $8(4+4)$ & $10(17.54 \%)$ & - \\
\hline $9(4+5)$ & $9(15.79 \%)$ & - \\
\hline $10(5+5)$ & $6(10.53 \%)$ & - \\
\hline \multicolumn{3}{|l|}{ Plasma PSA (ng/ml) } \\
\hline Median (IQR) & $33.01(0.024-10,000)$ & $1.8(0.134-4.00)$ \\
\hline$\leq 4$ & $16(28.07 \%)$ & $29(100 \%)$ \\
\hline $5-20$ & $9(15.79 \%)$ & $0(0.00 \%)$ \\
\hline $20-100$ & $14(24.56 \%)$ & $0(0.00 \%)$ \\
\hline$>100$ & $18(31.58 \%)$ & $0(0.00 \%)$ \\
\hline
\end{tabular}

tumours. Bishop et al. [22] recently reported that patients with CRPC resistant to enzalutamide showed elevated levels of PD-L1 in blood. The authors further concluded that PDL1 expression on tumour cells might be a mechanism of non-androgen receptor (AR)-driven resistance to enzalutamide.
There is a need for predictive biomarkers that can identify patients who will benefit most from anti-PD-1/PD-L1 immunotherapy. Our study and many others provide evidence of sPD-L1 as a prognostic biomarker for prostate cancer patients [36, 37]. Based on our results, we provide credible evidence that prostate cancer patients with higher Gleason scores are more 


\section{PD-1}

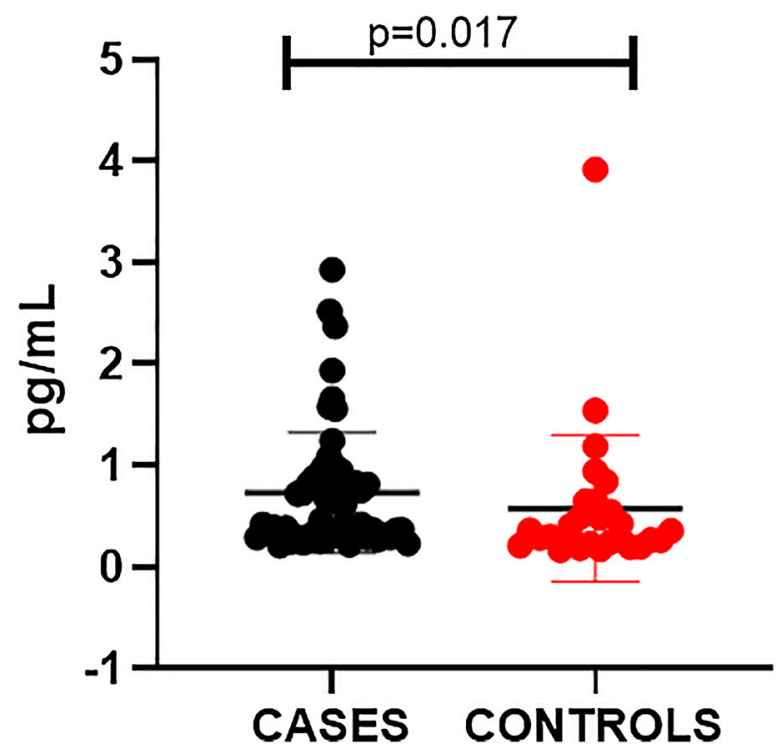

PD-L1

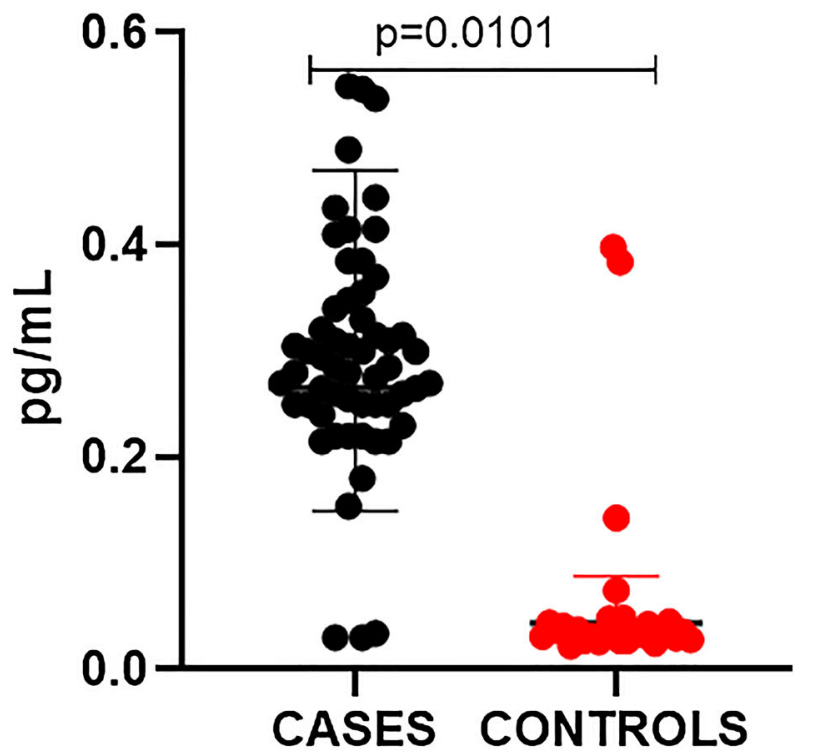

Fig. 1 Median concentrations of immune checkpoint molecules in prostate cancer patients and controls

Table 2 Association between immune checkpoint molecules and age, BMI, PSA, and Gleason score among prostate cancer patients and controls

\begin{tabular}{llllll}
\hline $\begin{array}{l}\text { Participants } \\
\text { Cases } \\
(\boldsymbol{n}=\mathbf{5 7})\end{array}$ & $\begin{array}{l}\text { Immune } \\
\text { checkpoint } \\
\text { molecules } \\
(\boldsymbol{n}=\mathbf{2 9})\end{array}$ & $\begin{array}{l}\text { Age } \boldsymbol{p} \text { value } \\
\text { correlation } \\
\text { coefficient }(\boldsymbol{r})\end{array}$ & $\begin{array}{l}\text { BMI } \boldsymbol{p} \text { value } \\
\text { correlation } \\
\text { coefficient }(\boldsymbol{r})\end{array}$ & $\begin{array}{l}\text { PSA } \boldsymbol{p} \text { value } \\
\text { correlation } \\
\text { coefficient }(\boldsymbol{r})\end{array}$ & $\begin{array}{l}\text { Gleason score } \boldsymbol{p} \text { value } \\
\text { correlation coefficient } \\
(\boldsymbol{r})\end{array}$ \\
\hline Cases & sPD-1 & $0.800(-0.0222)$ & $0.697(0.0396)$ & $0.758(0.0180)$ & $0.278(0.1425)$ \\
& sPD-L1 & $0.492(-0.0106)$ & $0.247(-0.1863)$ & $0.485(0.0657)$ & $0.014(0.3165)$ \\
Controls & sPD-1 & $0.397(-0.0465)$ & $0.087(0.3003)$ & $0.819(-0.0538)$ & N/A \\
& sPD-L1 & $0.039(-0.2776)$ & $0.056(0.2327)$ & 0.606 stata & N/A \\
\hline
\end{tabular}

likely to have higher levels of PD-L1 expression, with statistical significance. These patients are more likely to benefit from blocking the PD-1/ PD-L1 pathway.

This study faced several challenges which could have affected the final results, including the relatively low median age of the controls and the fact that only the plasma levels of PDL1/PD-1 were assessed and not tissue-based markers for comparison. The case-control study design was not able to determine the changes in checkpoint inhibitors over the course of treatments.

\section{CONCLUSION}

In this study we found that SPD-L1 was significantly associated with high Gleason scores. Our results shed more light on the possible role of 
sPD-L1 as a biomarker in prostate cancer. We recommend extensive prospective cohort studies to further validate these findings.

\section{ACKNOWLEDGEMENTS}

We want to thank all study participants at the Uganda Cancer Institute who consented to participate in this study.

Funding. Paul Katongole receives funding for doctoral studies from the African Development bank under Uganda Cancer Institute support training grant Project ID P-Z1-IB0-024 and is also supported by the Office of the Director, National Institutes of Health (OD), National Institute of Dental \& Craniofacial Research (NIDCR), National Institute of Neurological Disorders and Stroke (NINDS), National Heart, Lung, And Blood Institute (NHLBI), Fogarty International Center (FIC), National Institute on Minority Health and Health Disparities (NIMHD) grant number D43TW010132. In addition, D43TW010132 is funded in part (SJR) by the Division of Intramural Research, National Institute of Allergy and Infectious Diseases (NIAID). Its contents are solely the authors' responsibility and do not necessarily represent the supporting offices' official views. No funding or sponsorship was received for publication of this article.

Authorship. All named authors meet the International Committee of Medical Journal Editors (ICMJE) criteria for authorship for this article, take responsibility for the integrity of the work as a whole, and have given their approval for this version to be published.

Author Contributions. Paul Katongole, Obondo J Sande, and Nixon Niyonzima contributed to the conceptualization of the manuscript. Cynthia Nakimuli and Maxine Atuheirwe contributed to the running of the laboratory assays. Paul Katongole and Kenneth Ssebambulidde collected, cleaned, analyzed data and drafted manuscript. Henry Kajumbula, Samuel Kalungi, Moses Joloba, Steven J Reynolds,
Kenneth Ssebambulidde and Jackson Orem read and reviewed the manuscript information and data. All authors read and approved the final version of the manuscript.

Disclosures. Paul Katongole, Obondo J Sande, Steven J Reynolds, Moses Joloba, Henry Kajumbula, Samuel Kalungi, Kenneth Ssebambulidde, Cynthia Nakimuli, Maxine Atuheirwe, Jackson Orem and Nixon Niyonzima confirm that they have no conflict of interests to declare.

Compliance with Ethics Guidelines. This study obtained ethical approval from the School of Biomedical Sciences Higher Degrees Research and Ethics Committee (REF: SBS-HDREC- 779) and the Uganda National Council for Science and Technology. All study participants provided written informed consent before enrolment into the study.

Data Availability. All data files used in this manuscript article [and its supplementary information files] are available via fig share https://doi.org/10.6084/m9.figshare.14904870. v1.

Open Access. This article is licensed under a Creative Commons Attribution-NonCommercial 4.0 International License, which permits any non-commercial use, sharing, adaptation, distribution and reproduction in any medium or format, as long as you give appropriate credit to the original author(s) and the source, provide a link to the Creative Commons licence, and indicate if changes were made. The images or other third party material in this article are included in the article's Creative Commons licence, unless indicated otherwise in a credit line to the material. If material is not included in the article's Creative Commons licence and your intended use is not permitted by statutory regulation or exceeds the permitted use, you will need to obtain permission directly from the copyright holder. To view a copy of this licence, visit http://creativecommons.org/licenses/by$\mathrm{nc} / 4.0 /$. 


\section{REFERENCES}

1. James N, Lee N, Horton R. Announcing the Lancet Commission on Prostate Cancer. The Lancet. 2021;397(10288):1865-6.

2. Sung H, Ferlay J, Siegel RL, Laversanne M, Soerjomataram I, Jemal A, et al. Global cancer statistics 2020: GLOBOCAN estimates of incidence and mortality worldwide for 36 cancers in 185 countries. CA Cancer J Clin. 2021. https://doi.org/10. 3322/caac. 21660 .

3. Lewis DD, Cropp CD. The impact of African ancestry on prostate cancer disparities in the era of precision medicine. Genes. 2020;11(12):1471.

4. Koga Y, Song H, Chalmers ZR, Newberg J, Kim E, Carrot-Zhang J, et al. Genomic profiling of prostate cancers from men with African and European ancestry. Clin Cancer Res. 2020;26(17):4651-60.

5. Tan S-H, Petrovics G, Srivastava S. Prostate cancer genomics: recent advances and the prevailing underrepresentation from racial and ethnic minorities. Int J Mol Sci. 2018;19(4):1255.

6. Payne H, Mason M. Androgen deprivation therapy as adjuvant/neoadjuvant to radiotherapy for highrisk localised and locally advanced prostate cancer: recent developments. Br J Cancer. 2011;105(11): 1628-34.

7. Powers E, Karachaliou GS, Kao C, Harrison MR, Hoimes CJ, George DJ, et al. Novel therapies are changing treatment paradigms in metastatic prostate cancer. J Hematol Oncol. 2020. https://doi.org/ 10.1186/s13045-020-00978-z.

8. Teo MY, Rathkopf DE, Kantoff P. Treatment of advanced prostate cancer. Annu Rev Med. 2019;70(1):479-99.

9. Farkona S, Diamandis EP, Blasutig IM. Cancer immunotherapy: the beginning of the end of cancer? BMC Med. 2016. https://doi.org/10.1186/ s12916-016-0623-5.

10. Rusch T, Bayry J, Werner J, Shevchenko I, Bazhin AV. Immunotherapy as an option for cancer treatment. Arch Immunol Ther Exp. 2017;66(2):89-96.

11. Hargadon KM, Johnson CE, Williams CJ. Immune checkpoint blockade therapy for cancer: an overview of FDA-approved immune checkpoint inhibitors. Int Immunopharmacol. 2018;62:29-39.

12. Hwang WL, Pike LRG, Royce TJ, Mahal BA, Loeffler JS. Safety of combining radiotherapy with immunecheckpoint inhibition. Nat Rev Clin Oncol. 2018;15(8):477-94.
13. Xiong W, Gao Y, Wei W, Zhang J. Extracellular and nuclear PD-L1 in modulating cancer immunotherapy. Trends Cancer. 2021;7(9):837-46.

14. Zhu X, Lang J. Soluble PD-1 and PD-L1: predictive and prognostic significance in cancer. Oncotarget. 2017;8(57):97671.

15. Jain P, Jain C, Velcheti V. Role of immune-checkpoint inhibitors in lung cancer. Ther Adv Respir Dis. 2018;12:175346581775007.

16. Koppolu V, Rekha VV. Checkpoint immunotherapy by nivolumab for treatment of metastatic melanoma. J Cancer Res Ther. 2018;0(0):0.

17. Rosenberg JE, Hoffman-Censits J, Powles T, van der Heijden MS, Balar AV, Necchi A, et al. Atezolizumab in patients with locally advanced and metastatic urothelial carcinoma who have progressed following treatment with platinum-based chemotherapy: a single-arm, multicentre, phase 2 trial. The Lancet. 2016;387(10031):1909-20.

18. Handa S, Hans B, Goel S, Bashorun HO, Dovey Z, Tewari A. Immunotherapy in prostate cancer: current state and future perspectives. Ther Adv Urol. 2020;12:175628722095140.

19. Xian P, Ge D, Wu VJ, Patel A, Tang WW, Wu X, et al. PD-L1 instead of PD-1 status is associated with the clinical features in human primary prostate tumors. Am J Clin Exp Urol. 2019;7(3):159.

20. Gevensleben H, Dietrich D, Golletz C, Steiner S, Jung $\mathrm{M}$, Thiesler $\mathrm{T}$, et al. The immune checkpoint regulator PD-L1 is highly expressed in aggressive primary prostate cancer. Clin Cancer Res. 2016;22(8):1969-77.

21. Li Y, Huang Q, Zhou Y, He M, Chen J, Gao Y, et al. The clinicopathologic and prognostic significance of programmed cell death ligand 1 (PD-L1) expression in patients with prostate cancer: a systematic review and meta-analysis. Front Pharmacol. 2019;9: 1494.

22. Bishop JL, Sio A, Angeles A, Roberts ME, Azad AA, Chi KN, et al. PD-L1 is highly expressed in Enzalutamide resistant prostate cancer. Oncotarget. $2015 ; 6(1): 234$.

23. Gevensleben H, Holmes EE, Goltz D, Dietrich J, Sailer V, Ellinger J, et al. PD-L1 promoter methylation is a prognostic biomarker for biochemical recurrence-free survival in prostate cancer patients following radical prostatectomy. Oncotarget. 2016;7(48):79943.

24. Richter I, Jirasek T, Havlickova I, Curcikova R, Samal V, Dvorak J, et al. The expression of PD-L1 in 
patients with castrate prostate cancer treated with enzalutamide. Bone. 2018;29:87-9.

25. Han Y, Liu D, Li L. PD-1/PD-L1 pathway: current researches in cancer. Am J Cancer Res. 2020;10(3): 727.

26. Pardoll DM. The blockade of immune checkpoints in cancer immunotherapy. Nat Rev Cancer. 2012;12(4):252-64.

27. Brahmer JR, Drake CG, Wollner I, Powderly JD, Picus J, Sharfman WH, et al. Phase I study of singleagent anti-programmed death-1 (MDX-1106) in refractory solid tumors: safety, clinical activity, pharmacodynamics, and immunologic correlates. J Clin Oncol. 2010;28(19):3167.

28. Ostrand-Rosenberg S, Horn LA, Haile ST. The programmed death-1 immune-suppressive pathway: barrier to antitumor immunity. J Immunol. 2014;193(8):3835-41.

29. Nowicki TS, Hu-Lieskovan S, Ribas A. Mechanisms of resistance to PD-1 and PD-L1 blockade. Cancer J. 2018;24(1):47.

30. Lin Y-M, Sung W-W, Hsieh M-J, Tsai S-C, Lai H-W, Yang S-M, et al. High PD-L1 expression correlates with metastasis and poor prognosis in oral squamous cell carcinoma. PLoS ONE. 2015;10(11): e0142656.

31. Yi M, Niu M, Xu L, Luo S, Wu K. Regulation of PDL1 expression in the tumor microenvironment. J Hematol Oncol. 2021;14(1):1-13.
32. Yu W, Hua Y, Qiu H, Hao J, Zou K, Li Z, et al. PD-L1 promotes tumor growth and progression by activating WIP and $\beta$-catenin signaling pathways and predicts poor prognosis in lung cancer. Cell Death Dis. 2020;11(7):1-16.

33. Gevensleben H, Dietrich D, Golletz C, Steiner S, Jung M, Thiesler T, et al. The immune checkpoint regulator PD-L1 is highly expressed in aggressive primary prostate cancer. Clin Cancer Res. 2015;22(8):1969-77.

34. Li H, Wang Z, Zhang Y, Sun G, Ding B, Yan L, et al. The immune checkpoint regulator PDL1 is an independent prognostic biomarker for biochemical recurrence in prostate cancer patients following adjuvant hormonal therapy. J Cancer. 2019;10(14): 3102-11.

35. Topalian SL, Hodi FS, Brahmer JR, Gettinger SN, Smith DC, McDermott DF, et al. Safety, activity, and immune correlates of anti-PD-1 antibody in cancer. N Engl J Med. 2012;366(26):2443-54.

36. Yi M, Jiao D, Xu H, Liu Q, Zhao W, Han X, et al. Biomarkers for predicting efficacy of PD-1/PD-L1 inhibitors. Mol Cancer. 2018. https://doi.org/10. 1186/s12943-018-0864-3.

37. Shien K, Papadimitrakopoulou VA, Wistuba II. Predictive biomarkers of response to PD-1/PD-L1 immune checkpoint inhibitors in non-small cell lung cancer. Lung Cancer. 2016;99:79-87. 